\title{
Correction to: The pathogenicity of Th17 cells in autoimmune diseases
}

\author{
Keiko Yasuda ${ }^{1,2} \cdot$ Yusuke Takeuchi $^{1,3} \cdot$ Keiji Hirota $^{1}$ \\ Published online: 29 April 2019 \\ (C) Springer-Verlag GmbH Germany, part of Springer Nature 2019
}

\section{Correction to: Seminars in Immunopathology https://doi.org/10.1007/s00281-019-00733-8}

Unfortunately, an error occurred in the following passus of the article. The word "receptor" was missing in the sentence "Because T cells do not express GM-CSF receptor [41], GMCSF affects non-T cells."

The corrected text is given below.

GM-CSF, a key pathogenic cytokine in autoimmune tissue inflammation

GM-CSF is recently highlighted as the pathogenic cytokine of Th17 cells. The role of GM-CSF in EAE model was first reported in 2001, in which blockade of GM-CSF showed resistance to the EAE induction, but the critical source of GMCSF in immune cells was not investigated in detail [50]. There was the first report that among Th subsets infiltrating into the CNS after EAE induction, some of Th cells showed IL-17A+ GM-CSF+ double-positive producer [51]. The critical func- tion of IL-23 signaling directing encephalitogenic Th17 cells has been reported to drive GM-CSF production, which causes local tissue inflammation [41, 52]. Because $\mathrm{T}$ cells do not express GM-CSF receptor [41], GM-CSF affects non-T cells. GM-CSF first acts on CNS-infiltrating myeloid cells such as dendritic cells (DCs), monocytes, and macrophages which in turn secrete pro-inflammatory cytokines such as IL-6 and IL23 , both of which upregulate IL-23R expression, amplifying IL-23-mediated pathogenic circuit to directly cause neurological pathogenicity and establishing local tissue inflammation by recruiting inflammatory macrophages in the CNS $[51,53]$. GM-CSF also activates CCR2+ monocytes, monocytederived DCs, and microglia in the brain to produce IL-1 $\beta$ $[54,55]$. Since microglia have a potential to produce IL-23, they could participate in the IL-23-IL-17 immune axis in Th17 cell-mediated tissue inflammation [56].

The original article has been corrected.

Publisher's note Springer Nature remains neutral with regard to jurisdictional claims in published maps and institutional affiliations.

The online version of the original article can be found at https://doi.org/ 10.1007/s00281-019-00733-8

Keiji Hirota

hkeiji@infront.kyoto-u.ac.jp

1 Laboratory of Integrative Biological Science, Institute for Frontier Life and Medical Sciences, Kyoto University, Kyoto 606-8507, Japan

2 Department of Nephrology, Graduate School of Medicine, Osaka University, Osaka, Japan

3 Department of Rheumatology and Clinical Immunology, Graduate School of Medicine, Kyoto University, Kyoto, Japan 